\title{
Das Wesen der Heimat nach Kurt Stavenhagen
}

\author{
Andrzej GNiazDowsKI \\ Polish Academy of Sciences (Poland)
}

\begin{abstract}
The aim of the paper is to reconstruct the main premises of the notion of "homeland" as analyzed by Kurt Stavenhagen, the significant Latvian-German philosopher of the first half of the twentieth century. The paper points out the differences in Stavenhagen's approach to the notion of homeland between the first and second edition of his study, Heimat als Grundlage menschlicher Existenz (Homeland as the Basis of Human Existence) from 1939 and Heimat als Lebenssinn (Homeland as the Meaning of Life) from 1948. The paper informs about the biographical and political background of Stavenhagen's analysis and argues that his philosophical thought belongs to the so-called phenomenological movement. The thesis of the paper is that Stavenhagen's definition of the homeland as a kind of community presupposes Ferdinand Tönnies' distinction between community and society, which was fundamental for German sociology
\end{abstract}

of that time. The author argues that Stavenhagen follows Max Scheler's ideological misinterpretation of Tönnies' distinction in his "war writings" in that he contrasts two types of sociality: authentic and unauthentic. These types are characteristic for German and Anglo-American social relations, respectively. In this light, Stavenhagen's analysis turns out to be not so much a phenomenology but rather a mythology of the homeland. In reference to Leszek Kołakowski's book The Presence of Myth (1984), the paper presents the need for answering to the "phenomenon of the indifference of the world" as a proper meaning of Stavenhagen's mythology.

Keywords: Phenomenology, Homeland, Community, Society, Mythology, Political Philosophy, Capitalism, Exis-

tence

(c) Andrzej Gniazdowski; agniazdo@ifispan.edu.pl

Colloquium: New Philologies, Volume 6, Issue 1 (2021)

doi: 10.23963/cnp.2021.6.1.6

Stable URL: https://colloquium.aau.at/index.php/Colloquium/article/view/150

This work is licensed under a Creative Commons Attribution 4.0 International License (CC BY 4.0). 
Die philosophische Lyrik vom Philosophen und Hochschullehrer Kurt Stavenhagen (18841951), in welcher er das Wesen der Heimat besingt, klingt in polnischen Ohren mit unbestreitbar vertrauten Tönen aus. Als ein in Riga lebender und aufgrund des MolotovRibbentrop-Paktes aus dem heimatlich Lettland vertriebener baltischer Deutscher (Hiden \& Lane 2003), könnte er vielleicht auch seine emotionale Einstellung zum HeimatPhänomen in der diesbezüglichen polnischen Lyrik wiedererkennen. Das romantische Nationalepos Herr Thaddäus hat sein Verfasser Adam Mickiewicz mit den folgenden Worten inauguriert: „Litauen, du meine Heimat, du bist wie die Gesundheit. Nur wer diese verloren, weiß das Verlorene zu schätzen” (Mickiewicz 1976). Die Frage, ob die Heimat nichts anderes als Mythos ist, hat Mickiewicz mit dieser Invokation offensichtlich genauso negativ wie der heimwehkranke Stavenhagen beantwortet. Ihr Wesen scheint der baltische Pole wie sein deutscher Landsmann darin zu erblicken, dass sie nicht nur - mit Stavenhagen gesprochen - die "Grundlage menschlicher Existenz“ (Stavenhagen 1939), sondern auch der eigentliche „Lebenssinn“ des Menschen ist (Stavenhagen 1948).

Das Problem der Heimat und ihrer Bedeutung für das menschliche Leben hat Stavenhagen in einem Büchlein behandelt, das zweimal unter verschiedenen Titeln veröffentlicht wurde. Seine Vorkriegsauflage von 1939, welche in ihrem Titel die Heimat mit der Grundlage menschlicher Existenz identifizierte, unterscheidet sich von seiner zweiten Auflage, das unter dem Titel Heimat als Lebenssinn erschienen ist, auch inhaltlich. Im Vorwort der Nachkriegsauflage von 1948 hat Stavenhagen die Notwendigkeit dieser Unterschiede durch die „geänderten äußeren Verhältnisse“ erklärt, die einige Abschnitte von seinem Buch nicht mehr „zeitgemäß““ machte (Stavenhagen 1948, 3). Er hat auch darauf hingewiesen, dass er in seinen wissenschaftlichen Einsichten seitdem soweit weitergekommen ist, dass sein ganzes Buch, heute neu geschrieben, im Grunde entsprechend anders aussehen würde. Die Frage, die sich bezüglich beider Auflagen des Heimat-Buches von Stavenhagen von selbst stellt, betrifft einerseits den in ihnen unverändert identifizierten Wesensgehalt jenes Phänomens und andererseits die Motive der nach dem Kriege in seiner Beschreibung getroffenen Streichungen und Ergänzungen. In weiterer Folge betrifft diese Frage auch die eventuellen Korrekturen, welchen diese Wesensbeschreibung aus der Perspektive der gegenwärtigen, noch mehr geänderten äußeren Verhältnisse unterzogen werden sollte. 


\section{Die politische Phänomenologie von Kurt Stavenhagen}

Was die Eigentümlichkeit der Auffassung des Heimat-Phänomens von Stavenhagen besagt, ist in erster Linie der phänomenologische Hintergrund seiner Analysen. Im vergilbten Nekrolog aus einem alten Aktenordner, den der Verfasser des hiesigen Beitrags in einem Buch Stavenhagens gefunden hat, liest man, dass die meisten Untersuchungen des baltischen Deutschen der Phänomenologie der Gemeinschaftsformen galten. Gemäß diesem Nekrolog, der nach seinem Tod im Jahr 1951 vom Rektor der Georg-AugustUniversität zu Göttingen verfasst wurde, ist Stavenhagen, befreundet mit Max Scheler, im Austausch auch mit seinem Landsmann Nicolai Hartmann, philosophisch einen ganz selbständigen Weg gegangen. Das Denken von Stavenhagen, der 1908 in Göttingen mit einer Dissertation aus dem Bereich der Altgriechischen Philologie promovierte, sollte nach seinem Ausgang von den Griechen durch Erfahrung, Kampf und Leid eine Prägung von eigenartiger Kraft und Tiefe erfahren, um in der Reife als seinen Kern die Lehre der Verantwortung des Menschen zu enthüllen.

Als einen selbstständigen, mit den sogenannten Münchener und Göttinger phänomenologischen Kreisen verbundenen Phänomenologen, bezeichnete Stavenhagen auch der Philosophiehistoriker Herbert Spiegelberg. In seiner monumentalen Geschichte der phänomenologischen Bewegung hat Spiegelberg darauf aufmerksam gemacht, dass Stavenhagen erst als Vierzigjähriger begann, seine philosophischen Schriften zu publizieren (Spiegelberg 1965, 219). Inspiriert bei posthum erschienenen Fragmenten der Religionsphilosophie von Adolf Reinach hat Stavenhagen 1925 die ausführliche Studie Absolute Stellungnahmen. Eine ontologische Untersuchung über das Wesen der Religion veröffentlicht. Stavenhagen selbst hat in seinem Buch darauf hingewiesen, dass er sich in den dort enthaltenen Untersuchungen über die „persönlichen Stellungnahmen“ - und zwar nicht nur die absoluten, sondern auch die Stellungnahmen ,in der relativen Sphäre“ - mit den entsprechenden Forschungsergebnissen von Max Scheler, Edmund Husserl und Alexander Pfänder auseinandersetzen musste (Stavenhagen 1925, VII). Der Auseinandersetzung mit der „alten“, von ihren Gründern entwickelten Phänomenologie waren auch seine späten, nach dem Zweiten Weltkrieg entstandenen Studien gewidmet (Stavenhagen 1957). Nachdem er an die Universität Göttingen, seinem Studienort, zurückkehrte, hat Stavenhagen die Methode der nicht-Husserlschen Phänomenologie nach Spiegelberg besonders produktiv in Forschungsfeldern der philosophischen Anthropologie und der Ethik angewandt und seine frühere Untersuchungen über Person und Persönlichkeit in origineller, systematischer Form weiterentwickelt (Spiegelberg 1965, 220).

Neben Max Scheler mit seinen Kriegsschriften (Scheler 1915, Scheler 1916 und Scheler 2017), Edith Stein mit ihren Untersuchungen zur Sozial- und Staatsontologie (Stein 1922 und Stein 1925) sowie Dietrich von Hildebrand mit seinen ethisch-sozialen Schrif- 
ten und seiner antinazistischen, philosophischen Publizistik (Hildebrand 1916, Hildebrand 1922, Hildebrand 1930 und Hildebrand 1994), um nur die wichtigsten Repräsentanten jener Richtung innerhalb der phänomenologischen Bewegung zu nennen, hat Stavenhagen zur Entwicklung der soziologischen und politischen Anwendungsfähigkeit der Phänomenologie wesentlich beigetragen. Anders ausgedrückt: Der phänomenologische Hintergrund seiner theoretischen Zugangsweise zum Problem der Heimat macht es möglich, Stavenhagen als den Vertreter der soziologisch orientierten, „politischen Phänomenologie “ zu bezeichnen. ${ }^{1}$ Wie Spiegelberg bemerkt, konzentrierte sich der Rigaer Phänomenologe in den Dreißigerjahren zunehmend auf solche Probleme der Sozialphilosophie wie zum Beispiel das Wesen der Nation und des Volkes sowie das Phänomen der Heimat und der Solidarität, welche er mit der schöpferischen Anwendung der Studien von Alexander Pfänder und Moritz Geiger untersucht hat (Spiegelberg 1965, 220). Als die wichtigsten Ergebnisse jener Untersuchungen wurden im oben erwähnten Nachruf auf Stavenhagen, der seinerseits selbst als ein Zeitzeugnis betrachtet werden kann, das Buch Das Wesen der Nation von 1934 und eben das uns interessierende Werk Heimat als Lebenssinn genannt. Im ersten dieser Bücher sollte Stavenhagen, mit Rektor der Georgia Augusta gesprochen, „die glänzende Kritik des nationalen Massenwahns“ schreiben und im zweiten „die Bedeutung der echten Heimatbindung für die menschliche Existenz“ zeigen (Trillhaas 2014).

\section{Heimat als politisches Phänomen}

Was die Motive der Untersuchungen Stavenhagens über das Phänomen der Heimat anbelangt, waren sie zweifellos sehr eng mit seiner sozial-politischen Lage als Mitglied der deutschen Minderheit in Lettland verbunden. Es ist schwer, mit Spiegelberg nicht einig zu sein, dass dieselben Motive zum großen Teil auch hinter seiner Hinwendung zur Sozialphilosophie im Allgemeinen standen (Spiegelberg 1965, 220). Von seinen Göttinger Kommilitonen war Stavenhagen als der Heimatvertriebene in Erinnerung behalten, der sein ganzes philosophisches Leben der lettischen Heimat gewidmet hat. In dem bereits erwähnten Nachruf kann man lesen, dass ,er, dem wissenschaftliches Denken Lebenselement war“, nach seiner Promotion 1908 ,um seiner kurländischen Heimat willen“ auf die akademische Laufbahn verzichtet hat (Trillhaas 2014). Er sollte sich „unter das verpflich-

1 Der Darstellung der so von ihm bezeichneten Richtung innerhalb der phänomenologischen Bewegung hat der Verfasser dieses Textes das Buch gewidmet, wo versucht wird, die Weltanschauung der ersten Generation der Phänomenologen (Edmund Husserl, Max Scheler, Edith Stein, Ludwig Ferdinand Clauss, Dietrich Hildebrand) und die sowohl weltanschauliche als auch direkt politische Bedeutung ihrer soziologischen Analysen zu rekonstruieren (Gniazdowski 2015). Der vorliegende Vortrag kann als eine Fortsetzung des diesem Buch zugrundeliegenden Projektes verstanden werden. 
tende Gesetz seiner in der Existenz bedrohter Landmannschaft“ gestellt und ihr „später auch politisch und publizistisch in verantwortlichen Ämtern" gedient haben (Trillhaas 2014).

Einer der politischen Ausdrücke seiner Bereitschaft, der Heimat zu dienen war, dass Stavenhagen, zwölf Jahre nach seiner Heimkehr, im Jahr 1921 die Gründung der Rigaischen Deutschen Hochschule, des Herder-Instituts, in die Wege geleitet hat. Bis 1934 hatte er auch das Amt des Geschäftsführers der Deutschen Fraktion und des Deutschen Ausschusses der Parteien der deutschen Minderheit in Lettland inne, das er erst auf Druck nationalsozialistischer Kreise innerhalb jener Minderheit niederlegen musste (Tilitzki 2001, 806). Zu den publizistischen Beiträgen Stavenhagens zugunsten seiner kurländischen Landsmannschaft sind z.B. die während des Ersten Weltkrieges entstandenen Broschüren wie Stärke und Macht der Deutschen in den baltischen Provinzen (Stavenhagen 1915) sowie Die Kriegsziele des Grossrussentums und der Fremdvölker Russlands (Stavenhagen 1916) einzurechnen. Neben seinen dem Wesen der Nation und der Heimat gewidmeten Schriften hat er zum kulturellen und existenziell-politischen Selbstverständnis der baltischen Deutschen auch mit solchen Studien wie Diekonservativ-liberal-demokratische Staatsauffassungund Mitteleuropa (Stavenhagen 1927), Achtungals Solidaritätsgefühlund Grundlage der Gemeinschaften (Stavenhagen 1931) oder Kritische Gänge in die Volkstheorie (Stavenhagen 1936) beigetragen.

Diese starken politischen, die Untersuchung Stavenhagens über das Heimatphänomen leitenden Interessen kommen insbesondere ausdrücklich in der ersten Auflage seines Heimat-Buches zum Wort. Wie im Falle der anderen baltischen Deutschen ist die Innenpolitik der nach dem Ersten Weltkrieg neu entstandenen, unabhängigen Republik Lettland aufStavenhagens Missbilligung und Widerstand gestoßen (Purs 2012). Im Vorwort zu dieser Studie vom 20. September 1938 definierte Stavenhagen sein Buch als eine Hilfe denen, die „das Bedürfnis haben, sich über ein sie bedrängendes Existenzproblem ernsthaft Rechenschaft abzulegen“" (Stavenhagen 1939, 3). Selbst wenn er sich dort nicht explizit, aus verständlichen Gründen, auf die Bedrohung in der Existenz seiner eigenen lettischen Heimat bezog, lässt sich das Buch Heimat als Grundlage menschlicher Existenz als ein Versuch deuten, auf ihre schwierige, sozial-politische Lage aufmerksam zu machen. Um es zu tun, machte Stavenhagen zum porte parole seiner kurländischen Landsmannschaft, peinlicherweise zehn Tage vor dem Münchener Abkommen und dem Anschluss des Sudetenlandes an Deutschland, den Sudetendeutschen Gottfried Rothaker und sein Roman Dorf an der Grenze (Rothaker 1936). Mit Verweis auf dieses Buch, sowie auf den Roman Der Puchner von Wilhelm Pleyer (Pleyer 1934), machte Stavenhagen in einem nach dem Kriege gestrichenen Fragment der Einleitung darauf aufmerksam, dass das Problem der Heimat insbesondere schwer „auf vielen der unter fremdes Volkstum ver- 
streuten Gruppen“ lastet, die man als „die politisch Minderberechtigten oder gänzlich Enterbten, die nationalen Minderheiten nennt“(Stavenhagen 1939, 14).

In der Einleitung zu seinem Buch definierte Stavenhagen Heimat provisorisch als ein „endgültiges überindividuelles Zuhause“ (ibid.). In düsteren Farben schilderte er auch die politischen Hindernisse, auf welche der Wille einiger nationaler Minderheiten stößt, ein solches Zuhause zu behalten. Wie man dieser Einleitung entnehmen kann, musste er zu jenen direkten, politischen Hindernissen unter anderem die damalige Agrarreform in Lettland rechnen, die in der Enteignung des deutschen baltischen Adels von seinem Grundbesitz bestand (Purs 2012). „Zur seelischen Not der Verlassenheit und der lähmenden Wehrlosigkeit kommt die materielle“ (Stavenhagen 1939, 14). Neben der Ohnmacht des „nervenaufreibenden“, auf die Erhaltung des „täglich kleiner werdenden nationalen Besitzes“ abzielenden, passiven Widerstand, bestand diese Not im „Druck einer heute fast allmächtigen, das Steuer der Wirtschaft lenkenden Staatsmaschinerie“ und in den "gewaltsamen Assimilierungsversuche[n] und [der] Lockung zum Volksverrat" (ibid., 15). Für das Schlimmste angesichts der daraus entspringenden Alternative der Zukunft, ,in der Heimat wirtschaftlich verelenden oder sich aus der Heimat verdrängen lassen“, hielt Stavenhagen den Umstand, „dass die Jugend nicht mehr wurzelfest im Lande steht“ (ibid.). Er fragte: „Was für eine Zukunft hat eine Volksgruppe, wenn sie ihrer Jugend nicht mehr sicher sein kann?“ (ibid.).

\section{Die Heimat als Problem der Existenzphilosophie}

Das Heimat-Buch von Stavenhagen, in allen seinen Varianten, ist als Zeugnis der nicht nur politischen, sondern auch existenziellen Verzweiflung zu lesen. Die Frage, von welcher er persönlich ausgeht, mag zwar direkt politisch sein. Sie mag, als die Frage des Vertreters einer aus ihrer Heimat bald umzusiedelnden, nationalen Minderheit, lauten: „Was hat diese täglich neue Quälerei für einen Sinn? Geben wir wirklich etwas Unersetzliches auf, wenn wir, das Land der Väter verlassend, irgendwo in der Fremde ein menschenwürdiges Dasein suchen?“(Stavenhagen 1939, 15). Von der nicht abstreitbaren, sowohl politischen als auch wirkungsgeschichtlichen Relevanz dieser Frage abgesehen, betrachtete Stavenhagen sie dennoch als ein Beispiel dafür, wie nach der Bedeutung dessen, was menschliches Dasein betrifft, in einem „mehr äußerlichen Sinne“ zu fragen. Indessen erklärte er: „Hier soll die Frage im radikalsten Sinne gemeint sein: es soll nach der Bedeutung von Heimat für die menschliche Existenz gefragt werden. Es soll versucht werden, die Frage, welchen Sinn Heimat vom Standpunkt der Idee des Menschen aus, vom Standpunkt des Eigentlich-Menschlichen im Menschen aus hat, zu beantworten“ (ibid., 16). 
Worauf Stavenhagen fragte, war auf diese Weise nichts anderes als, mit Edmund Husserl aus seiner Krisis-Schrift gesprochen, „Lebensbedeutsamkeit“ (Husserl 1992, 3) von Heimat. Seine Frage war eine Frage aus dem Bereich der Existenzphilosophie, insofern als sie eine radikale Unterscheidung zwischen der eigentlichen und uneigentlichen, der Idee des Menschen nicht entsprechenden Existenz voraussetzte. Wie vor ihm der eingangs erwähnte Adam Mickiewicz hat er diese Unterscheidung mit der zwischen Gesundheit und Krankheit verglichen. In der ersten Auflage seines Buches begründete Stavenhagen die Möglichkeit der philosophischen Bestimmung einer „gesunden“, d.h. im Menschen eigentlich angelegten Existenzform, in Anknüpfung an die entsprechenden Ausführungen von Jaspers und Pfänder (Jaspers 1913; Pfänder 1933). Im Unterschied zu den auch gegenwärtigen Forschern aus dem Bereich der Tatsachenwissenschaften, die ein faktisches Verhältnis zwischen der „Ortsidentität" und „mentalen Gesundheit“ festzustellen versuchen (Lengen 2019, 121-149), war der phänomenologisch geschulte Stavenhagen einer damit verbundenen, „,methodischen Schwierigkeit“ durchaus bewusst. Er hat explizit zugegeben, dass die Frage, was Gesundheit ist, „sich nicht durch induktive Erfahrung feststellen“ lässt (Stavenhagen 1939, 16). Trotzdem wies er darauf hin, dass wir doch ein gewisses, wenn auch nicht genügend klares Bild davon haben, „wie körperliches Leben von Menschen aussteht, wenn es jener in ihm angelegten Vollkommenheitsmöglichkeit entspricht, die wir Gesundheit nennen“" (ibid.). Diesem Bild sollte nach Stavenhagen die „unmittelbare Anschauung“ zugrunde liegen, „die sich durch Wahrnehmung mehr oder weniger vollkommener Exemplaren gewinnen und in systematischer Interpretation verfeinern lässt“ (ibid.). Diese Feststellung hinsichtlich der Möglichkeit einer Bestimmung, was körperliche bzw. mentale Gesundheit ist, ließ sich seines Erachtens auf ein analoges Gebiet der Existenz des Menschen übertragen und die Frage, worin das eigentlichmenschliche Leben bestehen soll, auch als sinnvoll zu bestimmen.

Was nach Stavenhagen ein unklares Bild einer gesunden und dementsprechend kranken Existenz des gegenwärtigen Menschen lieferte, war die Beobachtung der nicht nur politischen, sondern auch ökonomischen und soziologischen Prozesse, welche die Lebensbedeutung der Heimat in Frage stellten. Im Unterschied zum direkten, gegen die tatsächliche Existenz der nationalen Minderheiten gerichteten Druck einer fremden Staatsmaschinerie, sollten diese Prozesse nach seiner Auffassung von allgemeiner und tiefgreifender Bedeutung sein. Den Grund des von ihnen in Gang gesetzten ökonomischen und soziologischen Wandels erblickte Stavenhagen in der durch den modernen Hochkapitalismus bedingten Einwicklung einer ,allumfassenden Arbeitsapparatur“, deren letzte und folgerichtigste Verwirklichung die Großstadt war (ibid., 5). Insofern als die großstädtische, auf ganz anderen als den „heimatlichen“ Prinzipien erbauten Lebensform des Menschen auch die Kleinstädte und das flache Land zunehmend beherrschte, wurde nach 
seiner Auffassung die Heimat selbst als eine eigentliche Art, auf die Welt bezogen zu sein, infolge jener hochkapitalistischen Entwicklung in ihrer grundlegenden, existenziellen Bedeutung gefährdet, wenn nicht überhaupt entwertet.

Aus der Perspektive der von Stavenhagen getroffenen Unterscheidung zwischen eigentlicher und uneigentlicher Existenz sollte großstädtisches Dasein als Dasein, „das nirgendwo hingehört und nirgendshin Bindungen hat" (ibid., 6), der Idee des Menschen radikal widersprechen. In seinem Buch ging Stavenhagen von der heftigen, gegen die großstädtische Lebensform gerichteten Philippika aus, welche die mit ihr zusammenhängende „innerliche Wurzellosigkeit des Menschen“ als den „Kern des Großstadtübels“ darstellte (ibid.). Großstädtisches Dasein sollte nach ihm die menschliche Existenz ihrer Eigentlichkeit berauben, da es als nomadisches Dasein in fortschreitender Weise den Menschen vom Menschen löste. Ein solches (großstädtisches) Wanderleben, dessen Symbol für Stavenhagen amerikanische Trailerstädte mit an Autos angehängte Wohnwägen waren, wie sie unter anderen Werner Herzog in seinem Stroszek porträtierte, sollte den Heimatsinn zerstören und sogar dicht besiedelte Länder in Niemandsländer verwandeln (ibid., 8).

Dem Großstadtleben und der mit ihr zusammenhängenden, entfremdeten Lebensform stellte Stavenhagen die im Miteinanderleben, in einer „persönlichen Umwelt“ bestehenden, eigentliche menschliche Existenz gegenüber. Sie sollte im „Heimatsinn als Verantwortungsgefühl für das Ganze“ gegründet sein, das, wie er betonte, nur „durch innere Bindung von Mensch zu Mensch entsteht" und das nur dann lebendig bleiben kann, wenn die Glieder jenes Ganzen „die Verantwortung auch wirklich tragen“ (ibid., 10). Wo diese zwischenmenschlichen, auf das Ganze gerichteten Bindungen in der „Dimension der Gleichzeitigkeit“ fehlen, bemerkte Stavenhagen, „können auch keine Bindungen von der Vergangenheit her und in die Zukunft da sein“ (ibid., 6). Heimatleben definierte er auf diese Weise als das auf dem Prinzip der Verantwortung gebaute Leben der aus einem eigenständigen Traditionskreis entstandenen, engeren Umweltgemeinschaft. „Menschentum, dem die Verantwortung für seine Stadt oder sein Land abgenommen wird, wird zum verantwortungslosen, Publikum', das sich von sich nur noch um die Achse seiner Privatexistenz dreht, die erforderlichen Pflichtleistungen für das Ganze aber von oben betreiben lässt“" (ibid., 10).

Was das Heimatbuch von Stavenhagen zu einem Zeugnis der nicht nur politischen, sondern auch existenziellen Verzweiflung macht, ist die von ihm gestellte Frage nach der Unausweichlichkeit der von ihm beobachteten „Entwicklung zur Heimatlosigkeit“ (ibid., 12). Nicht nur in der ersten, sondern auch in der zweiten Auflage seiner Studie wies er mit Zufriedenheit auf die damals zu Wort kommenden, in einer „Bewegung für Heimatkultur“ bestehenden Gegentendenzen hin. Neben der „wieder erwachenden Sehn- 
sucht nach Eingliederung in engere persönliche Gebilde, Heimatzentren“" (ibid., 12), betrachtete Stavenhagen als eindeutig positiv unter anderem die Erkenntnis, dass „Grund und Boden kein Spekulationsobjekt für die Börse sein darf“" (ibid.). In einem später gestrichenen Fragment der Vorkriegsauflage begrüßte er in diesem Kontext auch das 1933 erlassene Reichserbhofgesetz, in welchem, wie heute angenommen wird, die nationalsozialistische Blut-und-Boden Ideologie ihren vollen Ausdruck gefunden hat (Grundmann 1979). Die Frage, auf welche Stavenhagen nichtsdestotrotz immer wieder zurückkehrte, lautete: „Ist das berechtigt? Ist Heimat nicht doch eine sentimentale Angelegenheit, die es nicht wert ist, um ihretwillen gegen die angeblich eisernen Wirtschaftsgesetze anzurennen?" (Stavenhagen 1948, 13). 1938 kam das Bedenken hinzu: "Ist die Zustimmung zum Erbhofgesetz nicht das Ergebnis einer Stimmung, die verfliegen wird, wenn man die Härte der ökonomischen Gesetzmäßigkeiten beim Anrennen zu spüren bekommt?“ (Stavenhagen 1939, 14).

\section{Die Phänomenologie der Heimat}

Um diese schwierigen, aber seiner Meinung nach durchaus sinnvollen Fragen zu beantworten, machte Stavenhagen die Heimat ausdrücklich zum Gegenstand einer nach ihrem „Wesen“ suchenden Phänomenologie. Das Problem, worin „der Wert oder die Bedeutung von Heimat“ besteht, ob sie nicht bloß eine „nette Kindererinnerung“ oder, anders ausgedrückt, ein Mythos ist, behandelte er auch als das Problem ihrer Metaphysik und der an Scheler anknüpfenden materiellen Wertethik. Für ihre nicht-mythische Wirklichkeit hat der Rigaer Phänomenologe mit Verweis auf die oben erwähnten, im Menschen angelegten Möglichkeiten der Existenzvollendung argumentiert. Diese unklar geahnten und einer systematischen Interpretation bedürfenden Möglichkeiten sollten sich nach seiner Auffassung auch in einer bloßen Umwelt in „Fragen des Zweifels und der Auflehnung" melden: „Wozu bin ich überhaupt in dieser Umwelt? Warum soll ich eigentlich einem Personenumkreis, dem ich gleichgültig bin und der mir gleichgültig ist, ein Leben des Dienstes opfern? Warum soll ich das Beste, das ich zu geben habe, an Menschen verschleudern, die mir irgendein Zufall in den Weg geworfen hat?" (Stavenhagen 1948, 75).

Der eigentliche Ausgangspunkt der von Stavenhagen entwickelten, von diesen praktischen Fragen motivierten Phänomenologie der Heimat bildete eine anthropologische Feststellung bezüglich des Wesens des Menschen. Im Unterschied zu Helmuth Plessner, der im Rahmen seiner philosophischen Anthropologie von der These über die „exzentrische Positionalität" des menschlichen Lebewesens ausging (Plessner 1975, 288), war für Stavenhagen der Mensch als ein primär seelisches Wesen „in den Mittelpunkt seiner 
selbst gestellt" (Stavenhagen 1939, 17). In Anknüpfung an die Phänomenologin Hedwig Conrad-Martius und ihr Werk Metaphysische Gespräche hat er für sein Unterscheidungsmerkmal eine ,größere Eigenständigkeit" als irgendwelche andere Wesen gehalten (Conrad-Martius 1921, 26). Die Bedingung der Möglichkeit der philosophischen Erörterung des Heimatproblems, d.h. der Beantwortung der Frage nach der Bedeutung von Heimat für die eigentlich menschliche Existenz, hat Stavenhagen in der Anerkennung der metaphysisch-anthropologischen Tatsache erblickt, dass „der Mensch [...] nur in einer von sich aus, von innen nach außen geführten Existenz sich zu eigen gewinnen kann, nicht aber in einem wie ihm immer von außen her aufgedrängten Dasein, das seiner Seele zufällig ist und darum leer bleibt" (Stavenhagen 1939, 17-18).

Ausgehend von dieser anthropologischen Feststellung definierte Stavenhagen Heimat als „eine besondere Art, wie der Mensch auf die Gegenständlichkeiten der Welt bezogen und in ihnen verhaftet ist" (Stavenhagen 1948, 13). Die Besonderheit jener Beziehung sollte nach ihm in einer „Bodenständigkeit“ im Sinne eines "Zuhause-Seins“, d.h. eines solchen - nach Heidegger- „In-der-Welt-Seins“ bestehen, das ein gefühlsmäßiges Haften an den Gegenständlichkeiten einer bestimmten, „, mir nahestehenden und mich innerlich bergenden Umwelt" ist (Stavenhagen 1948, 16). Nach Stavenhagen sollte das Heimatliche keineswegs als Objektivität, sondern eher als eine durch den Wertnebensinn des Nahen und Trauten gekennzeichnete Beziehung bestehen. In anderen Worten: Sie soll nicht in einer räumlichen, sondern soziologischen Einheit bestehen. Deswegen war auch das Wesen der Heimat für ihn nicht etwa in einer Landschaft, sondern eher in einer die Heimat nach der Pascalschen „Logik des Herzens “ konstituierenden Gemeinschaft zu erblicken.

Mit Bezugnahme auf den Begriff der Gemeinschaft hat Stavenhagen seine Heimatlehre in den breiteren, theoretischen Kontext der phänomenologischen Soziologie gestellt. Konstitutiv für diese Zugangsweise zum Problem des Sozialen, zu deren Vertretern u.a. Max Scheler, Edith Stein, Dietrich von Hildebrand und Gerda Walther zu zählen sind, ist primär keineswegs die Intersubjektivitätstheorie Edmund Husserls oder der Satz Heideggers über das Dasein als „wesenhaft Mitsein“ (Heidegger 1993, 120) zu halten. Die phänomenologischen Analysen der sozialen Einheiten haben in erster Linie die von Ferdinand Tönnies vollzogene Unterscheidung zwischen „Gemeinschaft" und „Gesellschaft" bestimmt (Tönnies 2010). Bei den meisten Vertretern der phänomenologischen Bewegung ist diese von Tönnies als wertfrei gedachte Unterscheidung zu einer Gegenüberstellung der echten, „naturhaften und organischen“, und künstlichen, „rationalen und mechanischen “, auf dem Prinzip des Egoismus gegründeten Formen der sozialen Einheit geworden (Stein 2010, 111). Insbesondere während des Ersten Weltkriegs wurde diese Unterscheidung - wie z.B. in den sogenannten Kriegsschriften Schelers - mit der Gegen- 
überstellung von deutschen und englischen Arten der sozialen Beziehung gleichgesetzt, die einerseits von der „positiven Idee einer umfassenden Liebesgemeinschaft aller Geistund Vernunftwesen“ und andererseits vom positivistischen Ideal „einer bloß durch Interessensolidarität und Verträge geeinten Menschenmasse "bestimmt werden sollten (Scheler 1915, 116).

Aufgrund der Bezugnahme auf den Begriff der Gemeinschaft erwies sich die Frage nach dem Wesen der Heimat für Stavenhagen als gleichbedeutend mit der Frage nach der Konstitution der Gemeinschaft der ,in den Mittelpunkt" ihrer selbst gestellten, von „Innen“ nach „Außen“ existierenden Menschen (Stavenhagen 1939, 17-18). Die theoretischen Prämissen seiner Heimatlehre hat er ausführlich im Anhang zu seinem Buch besprochen, den er der analytischen Differenzierung der Aufbauelemente und Urformen sozialer Gebilde gewidmet hat. Obwohl diese Gebilde, nach der Bemerkung Stavenhagens, in ihrer historischen Wirklichkeit immer nur „Mischgebilde“ waren, hat er als ihre Urform die „geistige Gemeinschaft“ bzw. „Traditionskreis“ bezeichnet (Stavenhagen 1948, 117). Diese ursprüngliche Gemeinschaftsart sah er in der Allgemeingültigkeit bestimmter geistiger Inhalte in einer Gruppe von Personen gegründet, wie es z.B. in verschiedenem Maße innerhalb von Ständen, Stämmen, Völkern und Kulturkreisen, aber auch Familien und Vereinigungen der Fall ist (Stavenhagen 1948, 117).

Von den weit verstandenen, geistigen bzw. traditionellen Gemeinschaften, die er für die Grundlage aller anderen sozialen Gebilde hielt und die nach ihm auch selbständig auftreten konnten, unterschied Stavenhagen „Schicksalsgemeinschaften“ und "Gesellschaften“ (Stavenhagen 1948, 118). Innerhalb der ersteren differenzierte er zwischen „seelisch[en]“ und „situationsbedingten“ Schicksalsgemeinschaften, beispielsweise einerseits freundlich gesinnte Standes-, Landes- oder Volksgemeinschaften und andererseits pragmatische Verantwortungsgemeinschaften wie Nachbarschaften oder Polarexpeditionen. Die Grenzen zwischen diesen beiden Formen von Schicksalsgemeinschaften, wie auch jene zwischen Schicksalsgemeinschaften und „Interessen-,Gemeinschaften“" (zum Beispiel die amerikanische „Marktgemeinschaft“, der „Weltmarkt“ oder die „Aktiengesellschaft“), sollten nach Stavenhagen fließend sein (Stavenhagen 1948, 119).

In seinen Untersuchungen über das Wesen der Heimat ging Stavenhagen von der sozialphänomenologischen Unterscheidung zwischen der „Heimatgemeinschaft“ und dem „Heimatphänomen“ aus (Stavenhagen 1948, 21). Während die Heimatgemeinschaft für ihn ein im inneren Miteinandersein bestehendes soziologisches Ganzes war, bezeichnete er das Heimatphänomen als einen im Akt der Heimatliebe intendierten Gegenstand. Stavenhagen polemisierte gegen den üblichen Sprachgebrauch, nach welchem der gegenständliche Heimathintergrund, wie etwa die natürliche und künstliche Landschaft, Häuser, Straßen, Gärten, aber auch Sitte oder Sprechart, als ein konstituierendes Element der 
Heimat angesehen wird. Als das Primäre für die Heimat hat er „inneres Miteinandersein“, „Gemeinschaft“, oder „sogar irgendwie in einem besonders , hohen Grade“ Gemeinschaft" zu sein betrachtet (Stavenhagen 1948, 22). Ohne dieser ursprünglichen gemeinschaftlichen Dimension der individuellen und persönlichen „Beziehungen des Herzens“, so Stavenhagen, „wäre die seelische Wärme, die das Heimatganze ausstrahlt und die von den sie erlebenden Heimatgenossen zurückströmt, völlig unverständlich, denn sie fehlt allen anderen soziologischen Gebilden, z.B. den seit Tönnies sogenannten ,Gesellschaften“" (Stavenhagen 1948, 22).

Die von Kurt Stavenhagen vorgenommene phänomenologische Beschreibung des Heimatwesens ist wegen seiner keineswegs voraussetzungslosen Bezugnahme auf die Tönniesche Unterscheidung offensichtlich von vornherein als eine Art Mythologisierung der Heimat zu bezeichnen. Übrigens legte er über die mythologische Funktion seiner Beschreibung explizit Rechenschaft ab. Die Lebensbedeutsamkeit von Heimat definierte er genauso wie der andere Heimatvertriebene, Leszek Kołakowski, der in seinem Buch Die Gegenwärtigkeit des Mythos als Grund aller Versuche, sich mit den irrationalen, menschlichen Gemeinsamkeiten zu identifizieren, die Flucht vom „Phänomen der Gleichgültigkeit der Welt" interpretierte (Kołakowski 1984, 101). Auch Stavenhagen sah die irreduzible Lebensbedeutsamkeit der Heimat darin, dass sie uns „,vor der Gleichgültigkeit, die an sich zwischen Ich und Welt besteht, vor der seelische Kälte, die die Welt an sich erfüllt, vor dem nirgendwo innerlich hingehören“ bewahrt (Stavenhagen 1948, 85).

Wenn Helmuth Plessner in seinem Buch Grenzen der Gemeinschaft mit Recht feststellt, dass jedes Volk ,seinen Radikalismus“ hat (Plessner 1924, 21), kommt somit in der Phänomenologie der Heimat von Stavenhagen der in diesem Buch identifizierte „deutsche Radikalismus“ in seinem vollen Umfang zum Wort. Während der Radikalismus nach Plessner bei keinem Volk „den Sinn für die Wirklichkeit“ so sehr gefährdet, wie bei den Deutschen (ibid.), hat dennoch über die Radikalität der Frage nach der Heimat bei Stavenhagen sein Versuch entschieden, für ihre existenzielle und ethische Wirklichkeit sine ira et studio zu argumentieren. Stavenhagen wies ausdrücklich darauf hin, dass die Heimat als eine Umwelt- bzw. Schicksalsgemeinschaft „zu den Gegenständlichkeiten gehört, deren Gehalt immer nur bis zu einem gewissen Grade verwirklicht ist“ (Stavenhagen 1948, 17). Indem sie nach seiner Auffassung, wie alles irdische Sein, ,immer etwas Fragmentarisches, Unvollendetes“ war, hat er sie als „zum Teil Wirklichkeit“ und „zum Teil Ideal“, als einen „Grenzbegriff“ bezeichnet (ibid.). Die Frage nach einer „absoluten Gemeinschaft“, d.h. nach einem „schlechthinnigen Geborgensein und letztem Aufgehobensein“, wenn auch gleichfalls sinnvoll, hat Stavenhagen dagegen als die „Zentralfrage der Religionsphilosophie" betrachtet. 


\section{Schlusswort}

Zum Schluss sei darauf hingewiesen, dass damit die Heimat in ihrem phänomenalen Selbst erscheinen kann, eine bestimmte - mit Husserl $(1992,82)$ gesprochen - „Einstellungsänderung“ nötig war. „In der Alltäglichkeit“, schrieb Stavenhagen, ,ist der Heimatwert der Traulich-Bergenden wie verhüllt und im Gewöhnlichen und Banalen untergegangen“" (Stavenhagen 1948, 20). Um den Heimathintergrund aus seiner Stummheit zu erlösen und die Heimat zu schätzen zu wissen, bedarf es in seiner Auffassung „eines gewissen feiertäglichen Aufschwunges der Seele" (Stavenhagen 1948, 20). Nicht anders als Mickiewicz, der in Herr Thaddäus auf die Unumgänglichkeit des Verlustes als auf die Bedingung der Selbstgegebenheit der Heimat verwies, hat Stavenhagen diese Bedingung „in der Heimatliebe und in den Erscheinungsformen dieser Liebe, im Heimweh, in der Wiedersehensfreude und im Abschiedsschmerz" erblickt (Stavenhagen 1948, 21). Die Frage, inwieweit seine Untersuchungen über die Lebensbedeutsamkeit der Heimat unverändert auch heute einen Widerhall finden können, erweist sich im Grunde als dieselbe Frage, wie die nach der Lebensbedeutsamkeit unserer „rationalen“ Strategien - wie Leszek Kołakowski feststellt - die Erfahrung der „Gleichgültigkeit der Welt“ vor uns selbst verheimlichen (Kołakowski 1984, 103).

\section{Literatur}

Conrad-Martius, Hedwig. 1921. Metaphysische Gespräche. Halle: Max Niemeyer Verlag.

Gniazdowski, Andrzej. 2015. Antynomie radykalizmu. Fenomenologia polityczna $w$ Niemczech 1914-1933. Warszawa: Wydawnictwo IFiS PAN.

Grundmann, Friedrich. 1979. Agrarpolitik im Dritten Reich: Anspruch und Wirklichkeit des Reichserbhofgesetzes. Hamburg: Hoffmann und Campe.

Heidegger, Martin. 1993. Sein und Zeit. Tübingen: Max Niemeyer Verlag.

Hiden, John and Lane, Thomas. 2003. The Baltic States and the Outbreak of the Second World War. Cambridge: Cambridge University Press.

Hildebrand, Dietrich von. 1994. Memoiren und Aufsätze gegen den Nationalsozialismus 19331938. Mainz: Matthias-Grünewald-Verlag.

Hildebrand, Dietrich von. 1916. „Die Idee der sittlichen Handlung“. Jahrbuch für Philosophie und phänomenologische Forschung 3: 126-251.

Hildebrand, Dietrich von. 1922. „Sittlichkeit und ethische Werterkenntnis. Eine Untersuchung über ethische Strukturprobleme“. Jahrbuch für Philosophie und phänomenologische Forschung 5: 462-602.

Hildebrand, Dietrich von. 1930. Metaphysik der Gemeinschaft. Augsburg: Haas \& Grabherr. Husserl, Edmund. 1992. Die Krisis der europäischen Wissenschaften und die transzendentale Phänomenologie. Hamburg: Felix Meiner Verlag. 
Jaspers, Karl. 1913. Allgemeine Psychopathologie. Ein Leitfaden für Studierende, Ärzte und Psychologen. Berlin: Springer Verlag.

Kołakowski, Leszek. 1984. Die Gegenwärtigkeit des Mythos. Übersetzt von Peter Lachmann. München/Zürich: R. Piper \& Co.

Lengen, Charis. 2019. „Heimat und mentale Gesundheit: Wie place identity unsere Heimatgefühl und Wohlbefinden beeinflusst." In Heimat. Ein vielfailtiges Konstrukt, edited by Martina Hülz, Olaf Kühne, and Florian Weber, 121-149. Wiesbaden: Springer VS.

Mickiewicz, Adam. 1976. Pan Tadeusz. Versepos. Aus dem Polnischen nachgedichtet von Hermann Buddensieg. Berlin-Weimar: Eidos.

Pfänder, Alexander. 1933. Die Seele des Menschen. Versuch einer verstehenden Psychologie. Tübingen: Max Niemeyer Verlag.

Plessner, Helmuth. 1924. Grenzen der Gemeinschaft. Eine Kritikeinessozialen Radikalismus. Bonn: Friedrich Cohen.

Plessner, Helmuth. 1975. Die Stufen des Organischen und der Mensch. Einleitung in die philosophische Anthropologie. Berlin, New York: Walter de Gruyter.

Pleyer, Wilhelm. 1934. Der Puchner. Ein Grenzlandschicksal. München: Langen/Müller Verlag.

Purs, Aldis. 2012. Baltic Facades. Estonia, Latvia and Lithuania since 1945. London: Reaktion Books.

Rothaker, Gottfried. 1936. Dorf an der Grenze. Roman. München: Langen/Müller Verlag.

Scheler, Max. 1915. Genius des Krieges und der Deutsche Krieg. Leipzig: Verlag der Weißen Bücher.

Scheler, Max. 1916. Krieg und Aufbau. Leipzig: Verlag der Weißen Bücher.

Scheler, Max. 1917. Ursprünge des Deutschenhasses. Eine nationalpädagogische Erörterung. Leipzig: Verlag der Weißen Bücher.

Spiegelberg, Herbert. 1965. The Phenomenological Movement. A Historical Introduction. Volume One. The Hague: Martinus Nijhof.

Stavenhagen, Kurt. 1915. „Stärke und Macht der Deutschen in den baltischen Provinzen“. Grenzboten $74(4): 263-274$.

Stavenhagen, Kurt. 1916. Die Kriegsziele des Grossrussentums und der Fremdvölker Russlands. Charlottenburg: Ostlandverlag.

Stavenhagen, Kurt. 1925. Absolute Stellungnahmen. Eine ontologische Untersuchung über das Wesen der Religion. Erlangen: Verlag der Philosophischen Akademie.

Stavenhagen, Kurt. 1927. „Die konservativ-liberal-demokratische Staatsauffassung und Mitteleuropa“. Ostland 2: 51-62.

Stavenhagen, Kurt. 1931. Achtung als Solidaritätsgefübl und Grundlage von Gemeinschaften. Riga: G. Löffler.

Stavenhagen, Kurt. 1939. Heimat als Grundlage menscblicher Existenz. Göttingen: Vanderhoeck $\&$ Ruprecht.

Stavenhagen, Kurt. 1936. Kritische Gänge in die Volkstheorie. Riga: Plates.

Stavenhagen, Kurt. 1948. Heimat als Lebenssinn. Göttingen: Vanderhoeck \& Ruprecht.

Stavenhagen, Kurt. 1957. Person und Persönlichkeit. Untersuchungen zur Anthropologe und Ethik.

Göttingen: Vanderhoeck \& Ruprecht. 
Stein, Edith. 1922. „Individuum und Gemeinschaft“.Jahrbuch für Philosophie und phänomenologische Forschung 5: 116-283.

Stein, Edith. 1925. „Eine Untersuchung über den Staat“.Jahrbuch für Philosophie und phänomenologische Forschung 7: 1-123.

Tilitzki, Christian. 2001. Die deutsche Universitätsphilosophie in der Weimarer Republik und im Dritten Reich. Bd. 2. Berlin: Akademie Verlag.

Tönnies, Ferdinand. 2010. Gemeinschaft und Gesellschaft. Grundbegriffe der reinen Soziologie. Darmstadt: Wissenschaftliche Buchgesellschaft.

Trillhaas, Wolfgang. 2014. „Nachruf auf Kurt Stavenhagen“. Nachlass Wolfgang Trillhaas. Professor der Theologie 1905-1995. Göttingen: Niedersächsische Staats- und Universitätsbibliothek Göttingen. 